\title{
Analysis of humanoid appearances in human-robot interaction
}

\author{
Takayuki Kanda $^{1}$, Takahiro Miyashita ${ }^{1}$, Taku Osada $^{2}$, Yuji Haikawa ${ }^{2}$, Hiroshi Ishiguro ${ }^{1 \& 3}$ \\ ${ }^{1}$ ATR Intelligent Robotics and Communication Labs. $\quad{ }^{2} H o n d a ~ R \& D$ Co.,Ltd. \\ Kyoto, Japan \\ Saitama, Japan \\ ${ }^{3}$ Osaka University \\ Osaka, Japan
}

E-mail: kanda@atr.jp

\begin{abstract}
It is important to identify how much the appearance of a humanoid robot affects human behaviors toward it. We compared participants' impressions of and behaviors toward two real humanoid robots in simple human-robot interaction. These two robots have different appearances but are controlled to perform the same recorded utterances and motions, which are adjusted by using a motion capturing system. We conducted an experiment where 48 human participants participated. In the experiment, participants interacted with the two robots one by one and also with a human as a reference. As a result, we found that the different appearances did not affect the participants' verbal behaviors but did affect their non-verbal behaviors such as distance and delay of response. These differences are explained by two factors, impressions and attributions.
\end{abstract}

Index Terms - human-robot interaction; robot appearance; body movement analysis; humanoid robots.

\section{INTRODUCTION}

Over the past several years, many humanoid robots have been developed, and they can typically make sophisticated human-like expressions with their head, arms, and legs [1, 2]. We believe that humanoid robots will be suitable for our research on "communication robots" that behave as peerpartners to support daily human activities based on advanced interaction capabilities. As well as providing physical support, these robots will supply communication support such as routeguidance. To realize such a communication robot, it is important to identify its optimal appearance for HRI (humanrobot interaction).

Recent research in HCI (human-computer interaction) has highlighted the importance of robots as a new interface. Reaves \& Nass researched the role of computers as new interface media in the manner of TV and radio, and they proved that humans act toward computer interfaces (even a simple text-based interface) as if they were communicating with other humans [3]. Cassell et al. showed the importance of anthropomorphic expressions, such as arms and heads on embodied agents, for effective communication with humans [4]. Cory and Cynthia compared a robot and a computergraphic agent and found that the robot was suitable for communication about real-world objects [5].

As these research works suggest, the human-like bodies of humanoid robots enable humans to intuitively understand their gestures and cause people to unconsciously behave as if

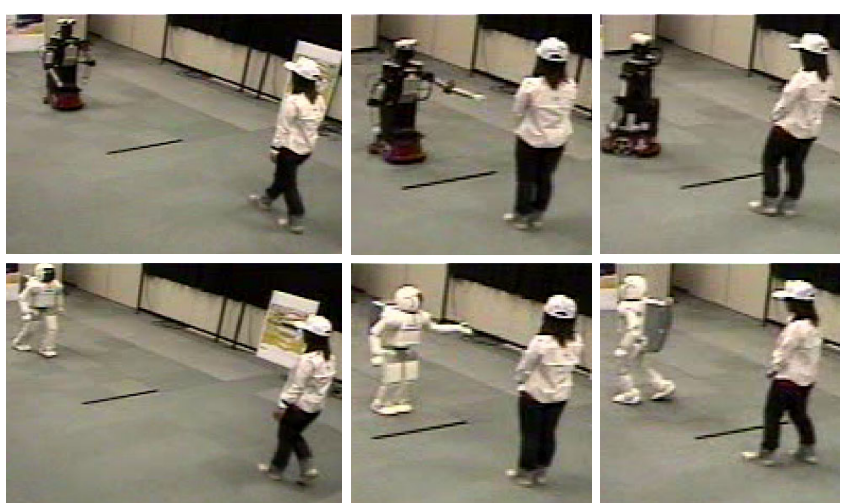

Figure 1: Robovie and ASIMO compared in the experiment left: approaching a participant (step 1), center: participants' talk (step 2) and robot's pointing at a poster (step 3), right: navigating the participant (step 4)

they were communicating with humans. That is, if a humanoid robot effectively uses its body, people will communicate naturally with it. This could allow robots to perform communicative tasks in human society such as route guides. Previous works in robotics also showed the effective usage of body properties in communication, such as head orientation based on real-time sensing by vision and audition [6] as well as utilization of facial emotions [7].

A few research works have been conducted to evaluate the appearance of robots in HRI. Goetz et al. compared the appearances of robot faces and found that a friendly face is appropriate for a playful task [8]. However, many robots were designed for interaction with humans, which inherently calls for friendly impressions. Rather, it is important to investigate the relationship between the amount of appearance difference and its effect on the interaction. In other words, it is important to identify to what extent the appearance of humanoid robots affects human behaviors toward robots. For example, some robots have biped-walking mechanisms while others have wheeled locomotion mechanisms. Similarly, there are many areas of differences, such as round-faced and rectangularfaced appearances, colored with either white or black. Such differences probably cause differences in subjective impressions. Key questions include whether these differences alter interaction, how much difference they make, and whether these differences are essential for the interaction.

One of the major difficulties in research on the appearances of humanoid robots is the question of control. Here, we mean controlling only one factor, such as biped- 
walking or wheeled locomotion, to identify the effect of each factor; this is a common experimentation method called "control" in psychology and HCI. It is very expensive to develop a humanoid robot, and financially impossible to develop several humanoid robots only for comparing appearances. Rather, it is realistic enough to compare existing humanoid robots as a case study [9] and make hypotheses on the effects of appearance. In addition, human beings can be a good reference of measurement for this comparison among humanoid robots. That is, by also comparing humanoid robots with human beings, the readers can fairly judge the importance of findings from the comparison among robots.

This paper reports experimental results on the effects of humanoid robots in simple interactions at first meeting (Figure 1). We compared two humanoid robots, ASIMO [2] and Robovie [10], and a human and found that not only the impressions but also attributions such as humanity affected the participants' non-verbal behaviors, although there were no differences found in their verbal behaviors.

\section{HUMANOID ROBOTS}

\section{A. Robovie}

Robovie [10] is a humanoid robot developed by the Intelligent Robotics and Communication Labs, ATR. They have developed communication robots, named Robovie, for the study of communication between individual humans as well as between humans and robots. Figure 2 (a) shows an overview of Robovie. It has a head, two arms, a body, and a wheeled type mobile base. On the head, it has two CCD cameras as eyes and a speaker as a mouth. The speaker can output recorded sound files installed on the internal control $\mathrm{PC}$ in the body. Its height and weight are $120 \mathrm{~cm}$ and $40 \mathrm{~kg}$, respectively. Degrees of freedom (DOFs) of the robot are as follows. It has 2 DOFs for the wheels, 3 DOFs for its neck, and 4 DOFs for each arm. Its motion can be controlled via a wireless LAN (IEEE 802.11b).

\section{B. $A S I M O$}

ASIMO [2] is a biped humanoid robot developed by HONDA. They have developed biped humanoid robots, P1, P2, P3 and ASIMO, in order to realize an autonomous architecture for humanoid robots. Figure 2 (b) shows an overview of ASIMO. It can walk and turn in any direction

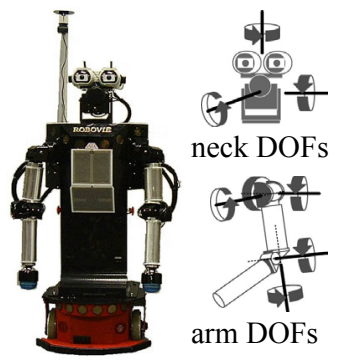

(a) Robovie

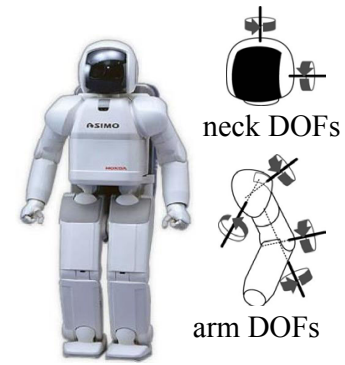

(b) ASIMO
Figure 2: Humanoid robots: Robovie and ASIMO. with its legs. On the head, it also has two cameras and a speaker. Its height and weight are $120 \mathrm{~cm}$ and $52 \mathrm{~kg}$, respectively. DOFs of ASIMO are as follows. It has 6 DOFs for each leg, 4 DOFs for each arm, 1 DOF for each wrist and hand, and 2 DOFs for its neck. Its motion, generated by a HONDA system, can be started and stopped via a wireless LAN.

\section{Robot motion}

We generated motions of the robots in accordance with the following principles. For ASIMO, we used the preset sample motions such as pointing motions for each arm, nodding and turning motions for the head, and walking and turning movements for the legs, and these motions were prepared by HONDA. For Robovie, we employed an optical motion capturing system to measure the motions of ASIMO in these sample motions and translated them into the motions of Robovie in order to achieve the same time period and loci of the motions for the head and each arm. The movement for the moving base was also adjusted to the same time period, directions and distances as those of ASIMO.

\section{Robot voice}

We recorded a human's voice (who is an experimenter in $\mathrm{H}$ condition) and used them for both Robovie and ASIMO, because our purpose was to compare the effect of different appearance of humanoid robot so we wanted to avoid making the experiment too complex. Of course, it is important future research to compare the effect of different voice and the balance between the appearance and quality or types of voice.

\section{EXPERIMENT}

\section{A. Participants}

The participants in our experiment were 48 university students (22 men and 26 women). Their average age was 20.6 years old.

\section{B. Conditions}

We conducted the experiments on simple interactions between the participants and each experimenter under the following three conditions.

Condition A: The experimenter is ASIMO.

Condition R: The experimenter is Robovie.

Condition $\mathbf{H}$ : The experimenter is a human

(Dr. Miyashita, one of the authors).

All participants interacted with an experimenter under each condition. We decided the order of the conditions randomly for each participant to counterbalance it.

\section{Environment}

Figure 3 shows the environment of the experiment. This is a room in our laboratory, in which the participants and each experimenter interacted. There were a black line at the center and four posters in the room. The posters were photographs of ancient structures of Kyoto, Japan. There were twelve IR cameras as the optical motion capturing system and a 


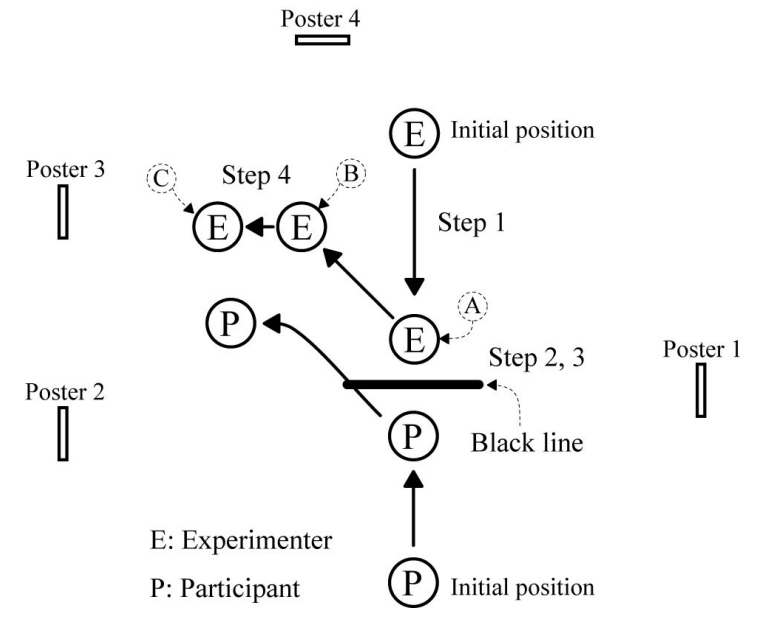

Figure 3: Environment and positions for experiments.

microphone placed around the environment for measuring the behaviors of participants and experimenters.

\section{Method}

As shown in Figures 1 and 3, each participant interacts with an experimenter, who moves in front of him/her. The details of the experiment are as follows:

\section{Step 1: The first meeting}

First of all, the participant is given an instruction at the initial position (Fig. 3): "The robot will come to the center of the room. Please go in front of the robot and greet it. Don't cross over the black line for your safety." Then, the experimenter moves forward at a constant speed and stops at a predetermined location ( $80 \mathrm{~cm}$ behind the black line). After stopping at this locomotion, the experimenter says, "Hello."

\section{Step 2: Participant's utterance to the experimenter}

While standing in front of the experimenter, the participant is given an instruction: "Please tell your name and the way to the laboratory to the robot." While the participant is telling this information, the experimenter nods when the utterance is a momentary paused (in the A and R conditions, this is controlled by an experimenter used in the $\mathrm{H}$ condition).

\section{Step 3: Conversation for orientation to the room}

Still in front of the experimenter, the participant is given the next instruction: "From now, the robot will explain this room to you. Please listen to it." Then, the experimenter says, "In Kyoto, there are many ancient structures. In this room, there are photographs of them." After this utterance, the experimenter turns its head to right, left and front to look around the room. Next, the experimenter says, "Please look at this," with the motion of turning its head to the left and pointing at poster 1 with left arm. When the motion is finished, the experimenter returns to a normal posture and says, "This is Kinkakuji Temple."

\section{Step 4: Navigation and conversation for guidance}

While still standing in front of the experimenter, the participant is given another instruction: "The robot will guide you to another place. Please follow it. From now, you can go across the black line." Then, the experimenter says, "Please follow me," and it turns clockwise 135 degrees with its moving base, either legs or wheels at point A. Next, the experimenter moves $1.4 \mathrm{~m}$ forward to point $\mathrm{B}$, turns counterclockwise 45 degrees at point $\mathrm{B}$, moves $0.5 \mathrm{~m}$ forward to point $\mathrm{C}$, and turns counterclockwise 90 degrees at point $\mathrm{C}$. Finally, the experimenter says, "Please look at this," with the motion of turning its head to the right and pointing at poster 3 with its right arm. When the motion is finished, the experimenter returns to the normal posture and says, "This is Ginkakuji Temple."

In the steps described above, time periods of the motions, velocities of the movement, and the positions and postures of

Table 1: Factor matrix (Varimax rotated).

These factors were interpreted by referring to factor loadings over 0.5 (shown in boldface) and named familiarity, novelty, safety, and activity factors.

\begin{tabular}{|l|l|r|r|r|r|}
\hline \multicolumn{2}{|c|}{} & Familiarity & Novelty & Safety & Activity \\
\hline Warm & Cold & $\mathbf{0 . 7 3 2}$ & 0.210 & 0.333 & 0.001 \\
\hline Accessible & Inaccessible & $\mathbf{0 . 7 0 7}$ & 0.056 & 0.177 & -0.221 \\
\hline Frank & Rigid & $\mathbf{0 . 6 7 5}$ & 0.179 & 0.150 & 0.120 \\
\hline Friendly & Unfriendly & $\mathbf{0 . 6 5 6}$ & 0.124 & 0.379 & -0.178 \\
\hline Cheerful & Lonely & $\mathbf{0 . 6 3 8}$ & 0.249 & 0.057 & 0.185 \\
\hline Light & Dark & $\mathbf{0 . 6 1 8}$ & 0.349 & 0.330 & 0.253 \\
\hline Humanlike & Mechanical & $\mathbf{0 . 5 9 6}$ & 0.132 & 0.134 & 0.323 \\
\hline Favorable & Unfavorable & $\mathbf{0 . 5 3 3}$ & 0.376 & 0.474 & 0.064 \\
\hline Showy & Quiet & $\mathbf{0 . 5 2 3}$ & 0.476 & -0.035 & 0.113 \\
\hline Light & Heavy & $\mathbf{0 . 5 1 2}$ & 0.141 & 0.057 & 0.191 \\
\hline Active & Passive & 0.456 & 0.201 & 0.445 & 0.159 \\
\hline Full & Empty & 0.432 & 0.389 & 0.004 & -0.006 \\
\hline Intelligent & Unintelligent & 0.062 & $\mathbf{0 . 7 4 2}$ & 0.217 & 0.117 \\
\hline Exciting & Dull & 0.409 & $\mathbf{0 . 6 4 7}$ & 0.281 & 0.206 \\
\hline Good & Bad & 0.398 & $\mathbf{0 . 5 9 9}$ & 0.500 & -0.033 \\
\hline New & Old & 0.435 & $\mathbf{0 . 5 9 5}$ & 0.162 & 0.180 \\
\hline Rich & Poor & 0.289 & $\mathbf{0 . 5 6 3}$ & 0.086 & 0.323 \\
\hline Likable & Dislikeable & 0.429 & $\mathbf{0 . 5 3 2}$ & 0.459 & -0.073 \\
\hline Interesting & Boring & 0.414 & $\mathbf{0 . 5 2}$ & 0.149 & -0.078 \\
\hline Sharp & Blunt & 0.261 & 0.497 & -0.110 & 0.417 \\
\hline Complex & Simple & -0.129 & 0.451 & -0.113 & 0.116 \\
\hline Clean & Dirty & 0.366 & 0.418 & 0.221 & 0.100 \\
\hline Happy & Unhappy & 0.308 & 0.412 & 0.228 & 0.187 \\
\hline Small & Large & -0.157 & -0.125 & $\mathbf{0 . 5 9 9}$ & 0.183 \\
\hline Kind & Cruel & 0.437 & -0.009 & $\mathbf{0 . 5 6 0}$ & -0.302 \\
\hline Distinct & Vague & 0.292 & 0.288 & $\mathbf{0 . 5 4 1}$ & 0.138 \\
\hline Safe & Dangerous & 0.354 & 0.107 & 0.494 & -0.153 \\
\hline Pleasant & Unpleasant & 0.399 & 0.130 & 0.493 & 0.209 \\
\hline Pretty & Ugly & 0.333 & 0.372 & 0.414 & -0.214 \\
\hline Altruistic & Selfish & 0.100 & 0.098 & 0.407 & -0.117 \\
\hline Calm & Agitated & 0.047 & 0.057 & 0.401 & -0.381 \\
\hline Rapid & Slow & 0.293 & 0.207 & -0.020 & $\mathbf{0 . 7 3 2}$ \\
\hline Quick & Slow & 0.318 & 0.204 & -0.146 & $\mathbf{0 . 6 0 7}$ \\
\hline Brave & Cowardly & -0.144 & 0.049 & 0.031 & $\mathbf{0 . 5 6 6}$ \\
\hline Robust & Delicate & 0.106 & 0.153 & -0.137 & $\mathbf{0 . 5 0 4}$ \\
\hline Strong & Weak & -0.004 & 0.028 & 0.121 & 0.414 \\
\hline
\end{tabular}




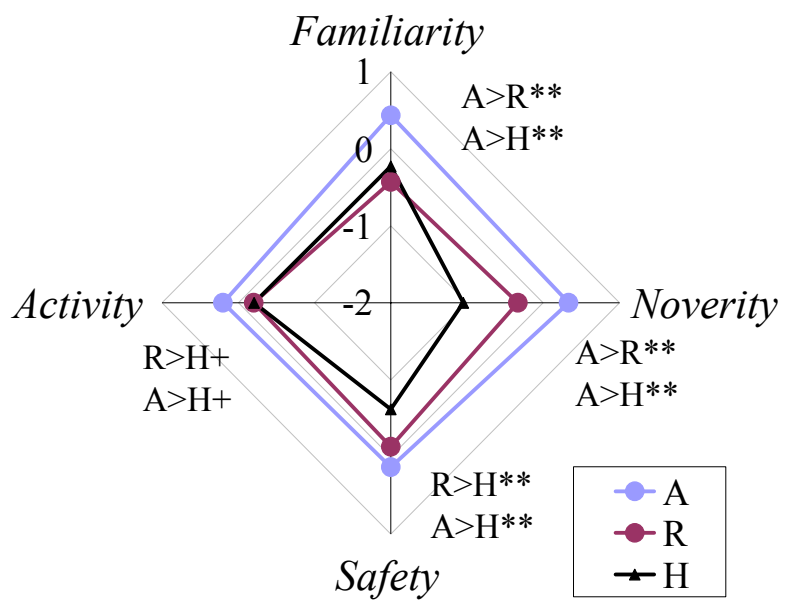

Figure 4: Comparison of impressions based on factor scores.

the robots were generated in accordance with the principle described in section II-C. The utterances of the robots were sound files recorded by the same human used as the experimenter under the $\mathrm{H}$ condition.

\section{E. Measurements}

We employed an optical motion capturing system to measure the participants' body movements. The motion capturing system consisted of 12 pairs of infrared cameras and infrared lights and markers that reflect infrared signals. These cameras were set around the room. The system calculates each marker's 3-D position from all camera images. The system has high resolution in both time $(120 \mathrm{~Hz})$ and space (accuracy of 1 $\mathrm{mm}$ in the room). We also measured their utterances with a microphone. At the end of the experiment under each condition, the participant was asked to answer a questionnaire for ratings by the SD (Semantic Differential) method. The questionnaire consisted of 36 adjective pairs shown in Table 1 in order to analyze the impressions of each experimenter.

\section{RESULTS}

\section{A. Impressions}

Following the method reported in [11], we conducted factor analysis on the SD ratings for the 36 adjective pairs. The Kaiser-Meyer-Olkin measure of sampling adequacy resulted in 0.835 , which is quite a good level. According to the differences in eigenvalues, we adopted a solution that consists of four factors. The cumulative proportion of the final solution was $48.9 \%$. The retrieved factor matrix was rotated by a Varimax method (shown in Table 1). These four factors were interpreted by referring to factor loadings and named Familiarity, Novelty, Safety, and Activity factors. Standardized factor scores were calculated to easily understand the results.

We compared the factor scores of each condition (Figure 4). ANOVA (analysis of variance) detected a significant difference in each of the four factors $(\mathrm{F}(2,143)=12.9 * *$, $37.5^{* *}, 10.3^{* *}, 3.5^{*}$, respectively, where “*” denotes

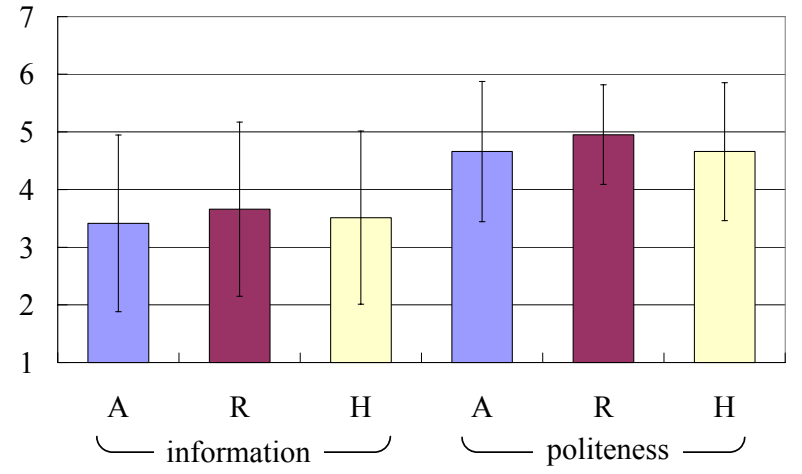

Figure 5: Comparison of participants' verbal response to the robots.

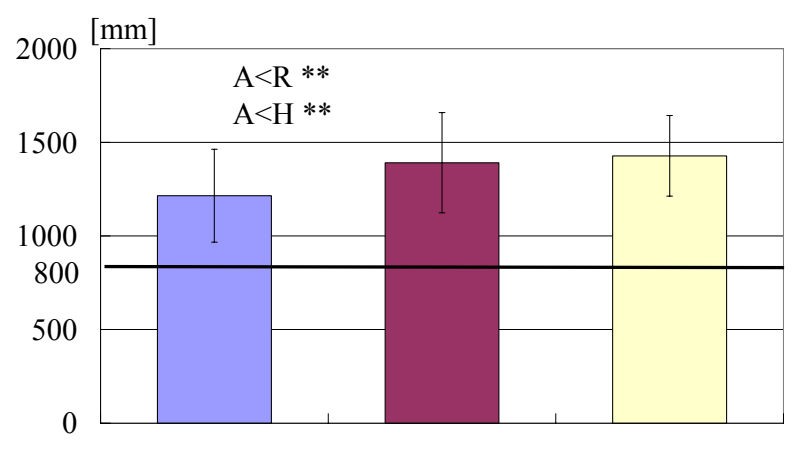

A

$\mathrm{R}$

$\mathrm{H}$

Figure 6: Distance between participants and experimenters at first time conversation.

significant difference at $\mathrm{p}<.05$ level, "***” denotes significant difference at $\mathrm{p}<.01$ level, and “+”denotes almost significant difference at $\mathrm{p}<.1$ level).

Then a Tukey HSD method was applied for multiple comparison among the conditions. As a result, it proved that the scores of A condition were significantly larger than those of $\mathrm{R}$ and $\mathrm{H}$ conditions for Familiarity at $\mathrm{p}<.01$ level (we denote this as $\left.\mathrm{A}>\mathrm{R}^{* *}, \mathrm{~A}>\mathrm{H}^{* *}\right)$. There are significant differences of $A>R^{* *}, A>H^{* *}$ for Novelty, $A>H^{* *}, \mathrm{R}>\mathrm{H}^{*}$ for Safety, and $A>R+(p=.061), A>H+(p=.057)$ for Activity. To summarize the results on subjective impressions, ASIMO received better subjective impressions than did Robovie or the human.

\section{B. Verbal responses}

The next analysis is on the participants' verbal behaviors toward the robots. At step 2 of the experiment (explained in Section 3), participants were requested to give their name and describe the way to the laboratory. We compared these utterances. Due to recording failure, 7 participants' data were omitted from the analysis, so we analyzed 123 items of utterance covering three observations of about 41 participants.

A third person scored these 123 utterances only by listening to the utterances, not knowing the experiment conditions such as to which experimenter the participants were talking. This evaluator used two measurement scales: the amount of information contained in the utterance and the 


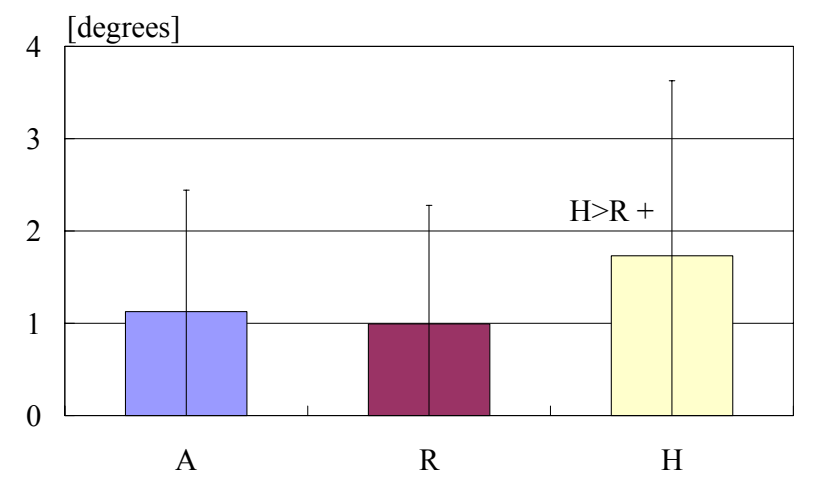

Figure 7: Degree of participants' waist angle at bowing as greeting.

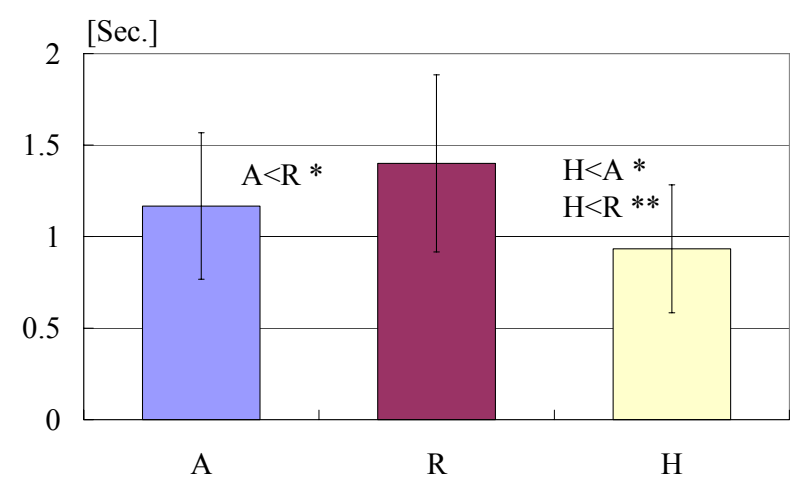

Figure 8: Participants' delay time of vocal-response to greeting.

politeness of the utterance. The scores were given on a 1-to-7 scale, where 7 represents the most positive, 4 represents the average, and 1 represents most negative impression (Figure 5). (In figures in the paper, colored bars represent average and vertical lines represent standard deviation. That is, the ranges of average $\pm 1 \sigma$ are denoted by the vertical lines.) As a result, ANOVA showed no significant difference among the three conditions (information: $\mathrm{F}(2,122)=0.269$, politeness: $F(2,122)=0.960)$. That is, the participants gave the same amount of information with the same politeness to ASIMO, Robovie, and a human.

\section{Non-verbal responses during conversation}

The motions and utterances of the robots, Robovie and ASIMO, were completely controlled so that both of them moved their upper torso in the same way. The human also behaved similarly to the robots. The participants' non-verbal behaviors were analyzed by using the motion capturing system, but some of the data were incomplete and thus omitted from the analysis due to occlusion of the motion capturing system.

Figure 6 shows a comparison of talking distance at experiment step 1 . The valid number of data for the analysis is 138 (46 participants). ANOVA proved a significant difference among conditions $\left(\mathrm{F}(2,137)=28.77^{* *}\right)$. The multiple

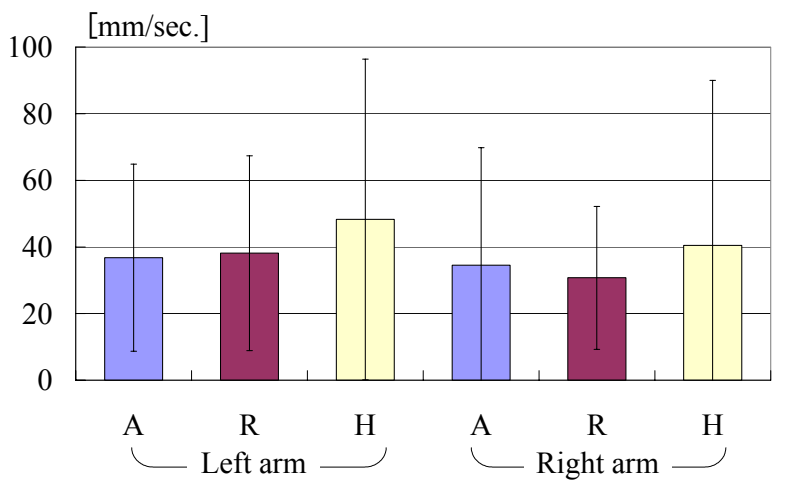

Figure 9: Amount of each arm's movements when participants were talking.

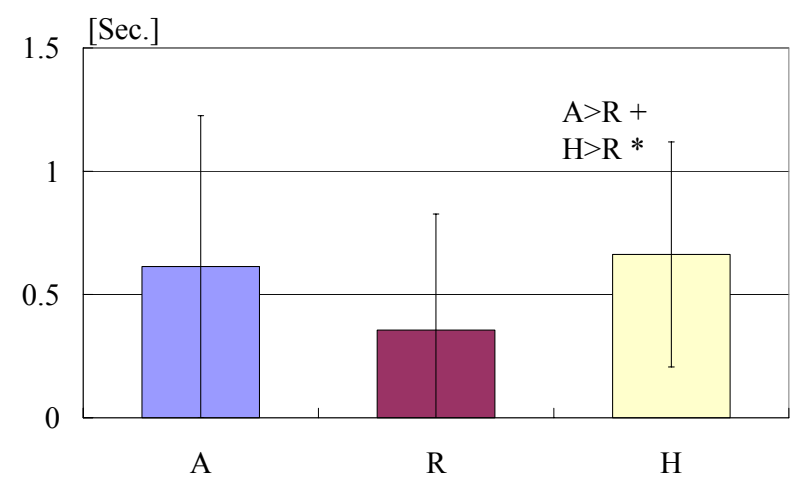

Figure 10: Participants' delay time of gaze-response to pointing.

comparison with Tukey HSD showed the results of multiple comparisons as $\mathrm{A}<\mathrm{R}^{*}$ and $\mathrm{A}<\mathrm{H}^{*}$. That is, the participants tended to stand closer to ASIMO than to Robovie or the human. The black line in Figure 6 represents the position of the black line on the room's floor. A few participants in A condition stood very close to the black line, so if there were no black line, perhaps they would have stood closer to ASIMO (although this would have been somewhat unsafe).

Next, we analyzed participants' response to the greeting from robots. At experiment step 1, robots said "hello," and the participant was, in advance, requested to respond to the greeting. The first comparison is based on the degree to which the participants bent their waist in bowing during the greeting, and 134 data items were analyzed (Figure 7). As a result of ANOVA, there were almost significant differences among conditions $(\mathrm{F}(2,133)=2.936, \mathrm{p}=.056)$. Tukey HSD also indicated an almost significant difference $\mathrm{H}>\mathrm{R}+(\mathrm{p}=.061)$. This suggests that participants would more deeply bow to the human than to Robovie.

The second comparison was on the delay time of the vocal response to "hello." Due to recording failures, 7 participants' data were omitted from the analysis, so 123 data items were analyzed (Figure 8). ANOVA proved significant difference among conditions $(\mathrm{F}(2,122)=12.852, \mathrm{p}<.01)$. Tukey HSD also showed the significant differences $\mathrm{H}<\mathrm{A}^{*}, \mathrm{~A}<\mathrm{R}^{*}$, 


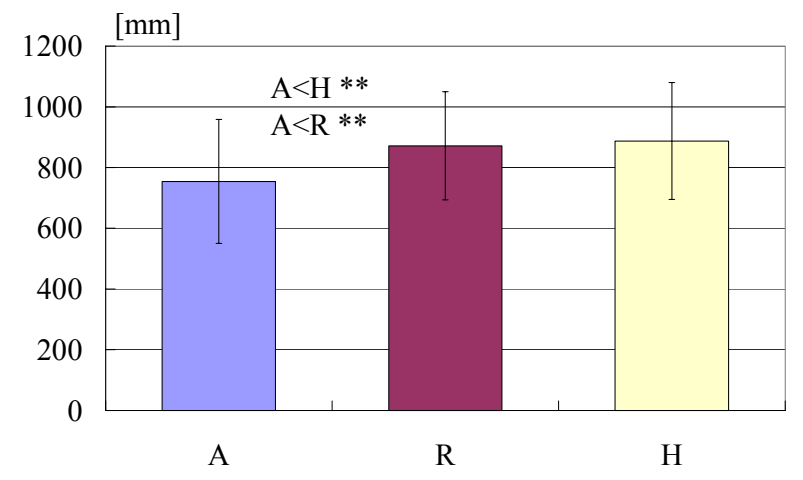

Figure 11: Distance between participants and experimenter during walking.

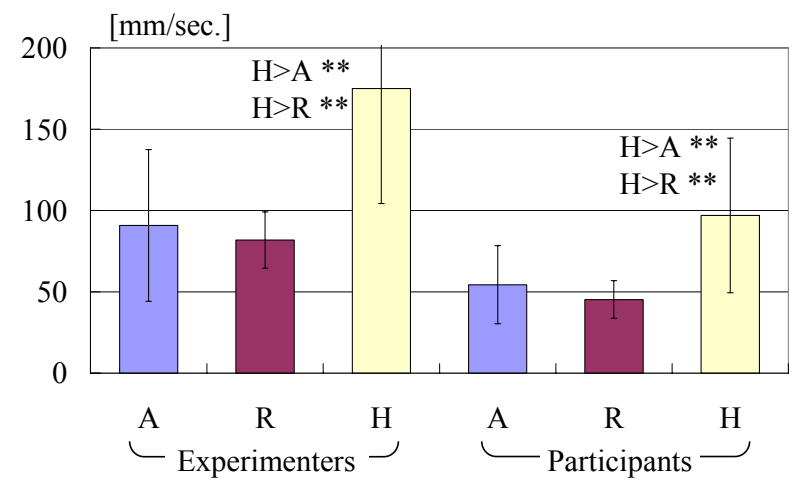

Figure 12: Speed of experimenters and participants during walking.

$\mathrm{H}<\mathrm{R}^{* *}$. To summarize the results, participants more rapidly replied to humans than to other robots, and they more rapidly replied to ASIMO than to Robovie.

To verify whether the amount of each participant's arm gesture would change depending on the experimenters, we analyzed the amount of arm movements made while participants talked to the robots at experiment step 2. Figure 9 shows the amount of each arm's movement per second. For each arm, ANOVA was applied, but no significant difference was found (left arm: $F(2,119)=1.203, p=.304$, right arm: $\mathrm{F}(2,117)=.689, \mathrm{p}=.504)$.

Figure 10 shows a comparison of the delay time of participants' response to the experimenters' pointing. At experiment step 3, experimenters pointed at a poster on the wall while talking about it. Most of the participants looked at the poster when the robot pointed to it. Here, 122 data items were analyzed for the participants looking at the poster as their head motion was successfully captured with the motion capturing system (number of data analyzed in each condition: A:39, R:43, H:40). ANOVA proved a significant difference among conditions $(\mathrm{F}(2,121)=4.276, \mathrm{p}<.05)$. Tukey HSD also showed the differences of $\mathrm{A}>\mathrm{R}+(\mathrm{p}=.065)$ and $\mathrm{H}>\mathrm{R}^{*}$. It seemed that the participants most rapidly looked at the poster when Robovie did the pointing.
Table 2: Summary and hypotheses for results.

\begin{tabular}{|l|l|l|l|}
\hline & & \multicolumn{3}{|c|}{ Result } & Hypothesis \\
\hline \multirow{4}{*}{ Impression } & Familiarity & $\mathrm{A}>\mathrm{R}, \mathrm{H}^{* *}$ & Appearance \\
\cline { 2 - 3 } & Novelty & $\mathrm{A}>\mathrm{R}>\mathrm{H}^{* *}$ & \\
\cline { 2 - 3 } & Safety & $\mathrm{A}, \mathrm{R}>\mathrm{H}^{* *}$ & \\
\cline { 2 - 3 } & Activity & $\mathrm{A}>\mathrm{R}, \mathrm{H}+$ & \\
\hline \multirow{4}{*}{$\begin{array}{l}\text { Verbal } \\
\text { behavior }\end{array}$} & Information & n.s. & \\
\cline { 2 - 4 } Non-verbal & Politeness & n.s. & \\
\cline { 2 - 4 } & Talking Distance & $\mathrm{A}<\mathrm{H}, \mathrm{R}$ & Impressions \\
\cline { 2 - 4 } & Greeting motion & $\mathrm{H}>\mathrm{R}+$ & Authority \\
\cline { 2 - 4 } & Greeting delay & $\mathrm{H}<\mathrm{A}<\mathrm{R}^{* *}$ & Authority \& Impressions \\
\cline { 2 - 4 } & Gaze delay & $\mathrm{H}, \mathrm{A}>\mathrm{R}^{*}$ & Authority \& Impressions \\
\cline { 2 - 4 } & Arm movement & $\mathrm{n} . \mathrm{s}$. & \\
\hline \multirow{3}{*}{$\begin{array}{l}\text { Walking } \\
\text { behavior }\end{array}$} & Distance & $\mathrm{A}<\mathrm{H}, \mathrm{R}^{*}$ & Impressions \\
\cline { 2 - 4 } & Speed & $\mathrm{H}>\mathrm{A}, \mathrm{R}^{*}$ & (due to experimenters) \\
\hline
\end{tabular}

\section{Non-verbal behaviors during walking}

Participants were supposed to follow the experimenters at experiment step 4, who asked "please follow me" and turned around at point $\mathrm{A}$, moved through point $\mathrm{B}$, arrived at point $\mathrm{C}$, and turned around at that point. We analyzed participants' behaviors during these sequences.

The first analysis involves about human-robot distance. All 144 data items were successfully analyzed (Figure 11). ANOVA proved significant differences among conditions $(\mathrm{F}(2,143)=6.898, \mathrm{p}<.01)$. Tukey HSD showed the significant differences of $\mathrm{A}<\mathrm{H}^{* *}$ and $\mathrm{A}<\mathrm{R} * *$.

Second, we analyzed the speed of participants and experimenters (Figure 12). ANOVA proved significant differences among the experimenters' speeds $\left(\mathrm{F}(2,143)=50.778, \mathrm{p}<.01\right.$, Tukey HSD shows $\left.\mathrm{H}>\mathrm{A}^{* *}, \mathrm{H}>\mathrm{R} * *\right)$ and participants' speeds $(\mathrm{F}(2,143)=36.996, \mathrm{p}<.01$, Tukey HSD showed $\left.\mathrm{H}>\mathrm{A}^{* *}, \mathrm{H}>\mathrm{R}^{* *}\right)$. It unfortunately showed the lack of control for human locomotion compared to that of the two robots. The difference in participants' speed seems to be due to the speed of the robots and the human. (It should be mentioned that it is very difficult to imitate robots' speed because it is quite slow, particularly when they turn around.) To summarize the results, since there is no significant difference between ASIMO and Robovie, the participants' speed of following was apparently not affected by their appearances.

\section{DISCUSSIONS}

\section{A. Appearance, impressions, and behaviors}

Table 2 shows a summary of the experimental results and our hypotheses on the reasons for the differences. Regarding the impressions, ASIMO gave a better impression in most of the factors, and Robovie gave a better impression than the human in Novelty and Safety. Thus, participants' impressions of the human were worse than those of the two robots. We believe this was caused by the experimental control, in which the human, a stranger to the participants, approached them without a particularly welcoming attitude such as a smiling face, a casual introduction, or conversation about common interests. On the contrary, the participants seemed to accept the robots, which behaved in the same way 
as the human, and to enjoy interacting with the novel robot they saw for the first time.

The non-verbal behaviors seemed to be affected by the participants' impressions. For example, the distance during talking and walking show similar tendencies to familiarity. This is consistent with proximity theory in psychology, as proposed by Hall [12].

However, the human, who gave worse impressions, got better reactions than Robovie in some cases. In greeting delay analysis (Figure 8), the human got a more rapid response than the two robots. In greeting motion analysis (Figure 7), a similar trend was found. The gaze delay in pointing (Figure 10) has the opposite trend to the greeting delay. Our hypothesis for this opposite trend is as follows: participants tend to look at the human longer than the robots when they point at the poster so the gaze delay is longer, showing that the participants respect the human more than the two robots as a partner of conversation. In other words, if a participant respects the partner (the human or robots), they probably respond to the greeting rapidly and look at them when they point at the poster.

Contrary to these differences in non-verbal behaviors, there was no significant difference found in verbal behaviors. The amount of information and the politeness was same among the conditions. Similarly, there was no significant difference in the amount of gesture (Figure 9). That is, the difference in appearance mainly affected non-verbal behaviors that were performed unintentionally rather than verbal behaviors that were rather performed intentionally. This is probably because the conversation in the experiment was not so complicated. In our previous experiment on a routeguidance situation [13], we observed that human participants used different words to humans and to Robovie (for example, giving simple landmarks to Robovie).

To summarize the experimental result, we can model human behaviors to robots or humans as:

\section{Non-verbal behaviors $=\boldsymbol{f}($ Impressions, Attribution $)$}

where attribution includes "whether it is respected as the conversation partner." In this experiment, at least humanity (human or not) could provide such an attribution as being respected as the conversation partner, but it is not yet clear whether some non-human existence, such as robots with very human-like appearance or a machine-like but sophisticatedly designed appearance, could provide this attribute. This issue should be investigated in our future research.

\section{B. Effect of biped-walking for communication}

In the experiment, the locomotion mechanism (bipedwalking or wheeled) of the robot was also included in the comparison, but there is still no evidence for whether this directly affected non-verbal behaviors. It did seem to affect impressions such as Novelty, so it probably indirectly affected participants' non-verbal behaviors.

Meanwhile, some participants commented that the excessively slow locomotion of the robots made them difficult to follow. We believe this is one of the important but unsolved robotics research directions for develop the robot that is easily followed by humans. (However, a recent newspaper article reported that Honda developed a biped-walking mechanism that is as fast as human slow walks, which could be a pace that humans can easily follow).

\section{Limitations}

First, since our comparisons are based on a case study between two existing robots, Robovie and ASIMO, the generality of the robots is limited. It does not ensure whether the findings from the experiment can be applied to all other humanoid robots. We believe, however, that it is realistic enough setting and a good start for research on the appearances of humanoid robots.

Regarding the comparison with the human, his movements were not exactly the same as those of the robots, although he did his best. This is because we cannot perfectly control body movements and timings. Thus, perhaps the difference of movements as well as appearance could cause differences in the participants' behaviors. Even though there is some degree of difficulty in experimental control, we believe that important knowledge was found from the comparison with the human.

This experiment only involved a situation reflecting a first-time conversation. It seems that Novelty had larger effect on non-verbal behaviors than did the other factors, but novelty effects do not continue so long [14]. It is also important future work to investigate temporal changes in impressions and behaviors.

\section{CONCLUSION}

We compared participants' impressions and behaviors during simple interaction for two humanoid robots, Robovie and ASIMO, which have different appearances. The motions of these robots' were adjusted by using a motion capturing system so that both of them behaved in the same way. We analyzed participants' verbal and non-verbal behaviors during greeting, self introduction, robots' pointing at objects, and navigation in a room as well as their subjective impressions of the robots. For comparison, a human experimenter performed the same actions and utterances as the robots. As a result, this case study has provided concrete data on how differently participants behave toward these two robots and the human, which is explained by impressions and attributions. The differences found were not so large. However, since it depends on the usage of humanoid robots whether such a difference is essential, we believe that the experiment provided evidence for deciding whether a particular difference in appearance should be considered for a particular usage.

\section{ACKNOWLEDGEMENT}

This research was supported by the National Institute of Information and Communications Technology of Japan.

\section{REFERENCES}


[1] K. Hirai, M. Hirose, Y. Haikawa, and T. Takenaka, The development of the Honda humanoid robot. IEEE International Conference on Robotics and Automation (ICRA'98), pp. 1321-1326. 1998.

[2] Y. Sakagami, R. Watanabe, C. Aoyama, S. Matsunaga, N. Higaki, and K. Fujimura, The intelligent ASIMO; System overview and intergration, IEEE/RSJ Int. Conf. on Intelligent Robots and Systems (IROS'02), pp. 2478-2483, 2002

[3] B. Reeves and C. Nass, The media equation. 1996.

[4] J. Cassell, T. Bickmore, M. Billinghurst, L. Campbell, K. Chang, H. Vilhjalmsson, and H. Yan, Embodiment in conversational interfaces: Rea. Conference on Human Factors in Computing Systems (CHI'99), pp. 520-527, 1999.

[5] C. Kidd and C. Breazeal, Effect of a Robot on User Perceptions. IEEE/RSJ International Conference on Intelligent Robots and Systems (IROS'04), 2004.

[6] K. Nakadai, K. Hidai, H. Mizoguchi, H. G. Okuno, and H. Kitano, RealTime Auditory and Visual Multiple-Object Tracking for Robots. International Joint Conference on Artificial Intelligence (IJCAI'01), pp. 1425-1432, 2001.

[7] C. Breazeal and B. Scassellati, A context-dependent attention system for a social robot, International Joint Conference on Artificial Intelligence (IJCAI'99). pp. 1146-1151, 1999.
[8] J. Goetz, S. Kiesler, and A. Powers, Matching robot appearance and behavior to tasks to improve human-robot cooperation, IEEE Workshop on Robot and Human Interactive Communication (ROMAN'03), 2003.

[9] B. G. Glaser, andA. L. Strauss, Discovery of Grounded Theory: Strategies for Qualitative Research, Aldine De Gruyter, 1967.

[10] T. Kanda, H. Ishiguro, M. Imai, and T. Ono, Development and Evaluation of Interactive Humanoid Robots, Proceedings of the IEEE, Vol. 92, No. 11, pp. 1839-1850, 2004

[11] T. Kanda, H. Ishiguro, and T. Ishida, Psychological analysis on humanrobot interaction, IEEE International Conference on Robotics and Automation (ICRA'01), pp.4166-4173, 2001.

[12] E. T. Hall, The Hidden Dimension, Anchor Books, 1990.

[13] M. Kamasima, T. Kanda, M. Imai, T. Ono, D. Sakamoto, H. Ishiguro, and Y. Anzai, Embodied Cooperative Behaviors by an Autonomous Humanoid Robot, IEEE/RSJ International Conference on Intelligent Robots and Systems (IROS'04), pp.2506-2513, 2004.

[14] T. Kanda, T. Hirano, D. Eaton, and H. Ishiguro, Interactive Robots as Social Partners and Peer Tutors for Children: A Field Trial, Journal of Human Computer Interaction, Vol. 19, No. 1-2, pp. 61-84, 2004. 